\title{
COMPARATIVE ANALYSIS OF SOME EXISTING KINETIC MODELS WITH PROPOSED MODELS IN THE BIOSORPTION OF THREE HEAVY METALS IN A FLOW-BATCH REACTOR USING FIVE SELECTED MICRO-ORGANISMS
}

\author{
I. C. Nwidi ${ }^{1, *}$ and J. C. Agunwamba ${ }^{2}$ \\ 1,2 Department of Civil EngineEring, University of Nigeria, NSUKKA, ENUGU STATE. NIGERIA \\ E-mail addresses: ${ }^{1}$ chineke_nwidi@yahoo.com, ${ }^{2}$ jonah.agunwamba@unn.edu.ng
}

\begin{abstract}
The biosorption of three heavy metal ions namely; $\mathrm{Zn}^{2+}, \mathrm{Cu}^{2+}$ and $\mathrm{Mn}^{2+}$ using five microorganisms namely; Bacillus circulans, Pseudomonas aeruginosa, Staphylococcus xylosus, Streptomyces rimosus and Yeast (Saccharomyces sp.) were studied. In this paper, the effectiveness of six existing and two proposed kinetic models were compared using two statistical parameters namely; linear regression coefficient of correlation $\left(R^{2}\right)$ and average relative error (ARE\%) which were employed to study the performance of each model on the biosorption of the three heavy metals by the individual biosorbents. In terms of highest values of $\mathrm{R}^{2}$, first proposed model accounted for $46.7 \%$, Pseudo second-order kinetics model $40 \%$ while Elovich, Webber-Morris and second proposed kinetic models accounted for $6.7 \%$ respectively of the total results for biosorption of the three heavy metals by five selected microorganisms. But based on values of ARE\%, first proposed kinetic model accounted for $93.3 \%$ while pseudo second-order kinetic model accounted for $6.7 \%$ of the results for biosorption of the three heavy metals by the five microbes.
\end{abstract}

Keynotes: Heavy metals, Biosorption, Kinetics Models, Comparative analysis, Average Relative Error.

\section{INTRODOCTION}

Biosorption is a physiochemical process that occurs naturally in certain biomass which allows it to passively concentrate and bind contaminants onto its cellular structure [1]. Biosorption typically involves a combination of active and passive mechanisms, starting with the diffusion of the metal ion to the surface of the microbial cell [2]. The ability of microorganisms to interact with and accumulate a variety of metal ions has been well documented [3]. However, there are significant variations in metal uptake capacity among different genera, different species, and also different strains within a species [4]. Water is very important to human, plants and animals lives but it is also the world's most threatened essential resource. Some of the worst industrial pollution is contaminating the world's most vulnerable water resources [5]. Many industries such as metal plating facilities, mining operations and tanneries discharge waste containing heavy metal ions [6]. As pollutants, heavy metals were intensively studied due to their significance from the point of view of persistence and toxicity. These toxic metals can cause accumulative poisoning, cancer and brain damage to human when found above the tolerance levels [7]. The application of biosorption in continuous processes has received increasing attention from researchers because of its potential industrial roles [8]. In fact, the decision of whether to use batch or continuous processes is a function of hydraulic flow, physical characters of the biosorbent(s), the types of target pollutant(s), space availability, and invested capital. If the flow rate is low, a simple manual batch process is the most economical [9]. Many different types of process configurations, such as stirred tank reactors, up-flow or down-flow packed bed reactors, fluidized bed reactors, rotating contactors, trickle filters and air-lift reactors, have been proposed and investigated for their industrial practicality $[9,10]$. Most of these have been used in applications that employ living microorganisms for removal of metal contaminants from complex industrial wastewaters $[10,11]$. 
In other to understand the mechanisms of biosorption, emphasis will be on the kinetics of bisorption studies which give detailed information on adsorbate uptake rates and on rate-controlling steps such as external mass transfer, intraparticle mass transfer, and biosorptive reaction(s) [12]. Several models have been derived in attempts to quantitatively describe kinetic behaviour during the adsorption process, but each model has its own limitations [13] as contained in Table 1.

The two proposed equations were born out of general rate kinetic equation which were calibrated and verified to give the following two equations:

First proposed kinetic model:

$$
\mathrm{m}=0.9:\left(C_{0}{ }^{1-m}-C_{t}{ }^{1-m}\right)=(1-m) K t
$$

Second proposed kinetic model [20]:

$$
\alpha=0.525: q_{t}=K t^{\alpha}+C
$$

\section{MATERIALS AND METHODS}

In this study, the materials used are microorganisms comprising of gram positive, gram negative bacteria and algae which were grown on nutrient broth. The microbes were obtained from Department of Microbiology University of Nigeria, Nsukka while heavy metal solutions were prepared by dissolving metal copper nitrate, manganese sulphate and zinc chloride respectively $\left(\mathrm{Cu}\left(\mathrm{NO}_{3}\right)_{2} \cdot 3 \mathrm{H}_{2} \mathrm{O}, \mathrm{MnSO}_{4} \cdot 4 \mathrm{H}_{2} \mathrm{O}\right.$ and $\mathrm{ZnCl}_{2}$ ) in water to the required concentrations. The biosorbents that were used are: Staphylococcus xylosus, Bacillus circulans. Pseudomonas aeruginosa Streptomyces rimosus. Yeast (Saccharomyces sp.)

\subsection{Biosorption Experiments}

Wastewater reservoir was filled with a mixture of wastewater obtained from University of Nigeria waste stabilization pond and zinc chloride to obtain $50 \mathrm{mg} / \mathrm{l}$ concentration of $\mathrm{Zn}^{2+}$ in the solution. $2.5 \mathrm{~g}$ of biosorbents were introduced inside the five treatment units respectively. Each of the five treatment units had baffle to enhance mixing of the influent wastewater with the biosorbent. The hobs at the exit of the treatment units have sieves (in this case, whatman filter paper) to limit the quantity of biosorbents that leave the treatment units. The flows into the treatment units and out of the batch reactor/reservoir were controlled by flow controls. The experimental set up is as contained in Figure 1. During the 3-day period of operation, the reactors were monitored for 30 minutes, 2 hours, 24 hours, 48 hours and 72 hours respectively for metal ion concentration of the effluent solution and biosorption capacity (q).

The biosorption capacity or metal uptake was calculated using the following mass balance equation:

$$
q_{t}=\left(C_{0}-C_{t}\right) \frac{V}{M}
$$

In (3) $q_{t}$ is the biosorption capacity or metal uptake at time $\mathrm{t}(\mathrm{mg} / \mathrm{g}) ; \mathrm{C}_{\mathrm{o}}$ and $\mathrm{C}_{\mathrm{t}}$ are the initial metal ion concentration and metal ion concentrations at time $t$ in the solution $(\mathrm{mg} / \mathrm{l})$, respectively; $V$ is the solution

\begin{tabular}{|c|c|c|c|}
\hline $\mathrm{S} / \mathrm{N}$ & Expression & Equation form & Limitations \\
\hline 1 & $\begin{array}{l}\text { First-order kinetic rate } \\
\text { equation [14] }\end{array}$ & $-\ln \left(\frac{C_{t}}{C_{0}}\right)=k_{1} t$ & $\begin{array}{l}\text { Early applied second- order rate equation in } \\
\text { solid/liquid system }\end{array}$ \\
\hline 2 & $\begin{array}{l}\text { Second-order kinetic } \\
\text { rate equation [15] }\end{array}$ & $\frac{1}{C_{t}}=k t+\frac{1}{C_{0}}$ & $\begin{array}{l}\text { Early applied second- order rate equation in } \\
\text { solid/liquid system }\end{array}$ \\
\hline 3 & $\begin{array}{l}\text { Lagergren equation [16] } \\
\text { (Pseudo first-order } \\
\text { kinetic model) }\end{array}$ & $\log \left(q_{e}-q_{t}\right)=\log \left(q_{e}\right)-K_{1} t / 2.303$ & $\begin{array}{l}\text { Based on adsorption capacity and is linear } \\
\text { within the first few minutes. }\end{array}$ \\
\hline 4 & $\begin{array}{l}\text { Ho equation [15] } \\
\text { (Pseudo second-order } \\
\text { Kinetic model) }\end{array}$ & $\frac{t}{q_{t}}=\frac{1}{k_{2} q_{e}^{2}}+\frac{1}{q_{e}} t$ & $\begin{array}{l}\text { Based on adsorption capacity and so a } \\
\text { specific but different rate constant is } \\
\text { obtained for each change in system variable }\end{array}$ \\
\hline 5 & $\begin{array}{l}\text { Elovich kinetic model } \\
\text { [17] }\end{array}$ & $q_{t}=\left(\frac{1}{\beta}\right) \ln (\alpha \beta)+\left(\frac{1}{\beta}\right) \ln t$ & Chemisorption \\
\hline 6 & $\begin{array}{l}\text { Webber-Morris kinetic } \\
\text { equation [18] }\end{array}$ & $q_{t}=k t^{1 / 2}+C$ & Intraparticle-diffusion \\
\hline 7 & $\begin{array}{l}\text { General rate kinetic } \\
\text { equation [19] }\end{array}$ & $-\frac{d \lambda_{t}}{d t}=k \lambda_{t}^{n}$ & Non-fixed reaction order \\
\hline
\end{tabular}
volume (l); and $\mathrm{M}$ is the mass of biosorbent (g).

Table 1: Existing kinetic models 


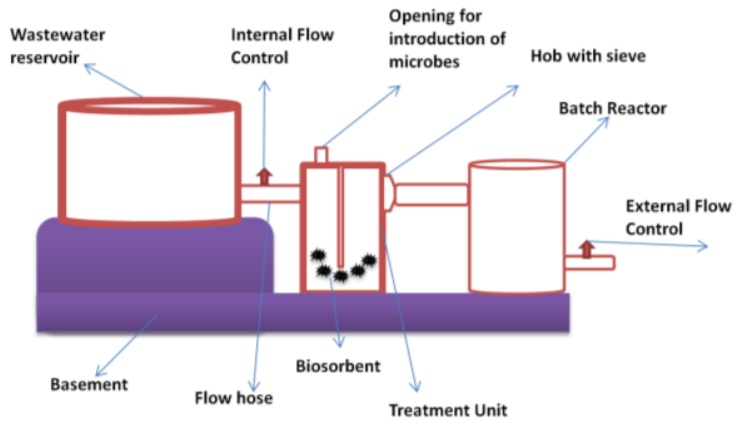

Figure 1: Section of the experimental set up

The $\mathrm{pH}$ of both reactors was kept constant with the aid of $0.1 \mathrm{~m} \mathrm{HCl}$ and $0.1 \mathrm{~m} \mathrm{NaOH}$. Room temperature was maintained throughout the experiments. The metal concentration of both the influents and effluents were obtained using a HI83200 Multiparameter spectrophotometer. The same experiment was repeated for $\mathrm{Cu}^{2+}$ and $\mathrm{Mn}^{2+}$.

\section{RESULTS AND DISCUSSIONS}

A comparative analysis was done on the six cited kinetic models in the literature as contained in Table 1 and the two proposed kinetics models to know which one of them could best accommodate different biosorption mechanisms.

Each of the cited kinetic models has one or more limitations with respect to the mechanisms of biosorption as contained in Table 1 . In this comparative analysis, two parameters were employed in the comparative analysis of the six existing kinetic models and the two proposed models. The two parameters are linear regression coefficient of correlation $\mathrm{R}^{2}$ and average relative error (ARE\%). The linear regression coefficient of correlations were calculated using the following representative of $\mathrm{x}$ and $\mathrm{y}$. ( $\mathrm{x}$ represent time abscissa, y represent ordinate)

$$
R .=\sqrt{\frac{\left(n \sum x y-\sum x \sum y\right)^{2}}{\left(n \sum x^{2}-\left(\sum x\right)^{2}\right)\left(n \sum y^{2}-\left(\sum y\right)^{2}\right)}}
$$

Similarly, average relative errors were calculated from the following equation:

$$
A R E=\frac{100}{N} \sum_{i=1}^{p}\left|\frac{X_{e, \text { meas }}-X_{e, \text { calc. }}}{X_{e, \text { meas. }}}\right|
$$

In (5) $X_{e}$, meas. is the measured variable, $X_{e}$, calc. is the calculated variable, $\mathrm{N}$ is the number of data points and $\sum$ is summation [21].

From Tables 2, 3 and 4, each cell contains the values for linear regression coefficient of correlation, average linear error and biosorption rate constants measured per minute. From Table 2, judging by least values of average relative error, the first proposed kinetic equation describes best the kinetics for biosorption of $\mathrm{Zn}^{2+}$ by Bacillus circulans, Pseudomonas aeruginosa, Streptomyces rimosus and Saccharomyces sp. While Pseudo second-order equation describes best the kinetics for biosorption of $\mathrm{Zn}^{2+}$ by Staphylococcus xylosus. This means that the whole mechanisms were accommodated fairly by both first proposed model and pseudo second-order model. But in terms of values of linear regression coefficient of correlation $R^{2}$, first proposed kinetic equation best described the kinetics for the biosorption of $\mathrm{Zn}^{2+}$ by Bacillus circulans and Pseudomonas aeruginosa, pseudo second-order kinetics model best described the kinetics for biosorptiuon of $\mathrm{Zn}^{2+}$ by Staphylococcus xylosus and Streptomyces rimosus and second proposed equation best described the kinetics for biosorption of $\mathrm{Zn}^{2+}$ by Saccharomyces sp.(Yeast).

\begin{tabular}{|c|c|c|c|c|c|c|c|c|}
\hline Microbe $\$$ K.Eq. & $\begin{array}{c}1^{\text {st }} \text { Order K. } \\
\text { Model R2 } \\
\text { ARE } \% \\
\text { Biosorption } \\
\text { Constant (K) }\end{array}$ & $\begin{array}{c}\text { 2nd Order K. } \\
\text { Model. R2 } \\
\text { ARE\% } \\
\text { Biosorption } \\
\text { Constant (K) }\end{array}$ & $\begin{array}{l}\text { Pseudo } 1^{\text {st }} \\
\text { Order K. } \\
\text { Model R² } \\
\text { ARE } \\
\text { Biosorption } \\
\text { Constant (K) }\end{array}$ & $\begin{array}{l}\text { Pseudo } 2^{\text {nd }} \\
\text { Order K. } \\
\text { Model. } \\
\text { RªRE } \\
\text { Biosorption } \\
\text { Constant (K) }\end{array}$ & $\begin{array}{c}\text { Elovich K. } \\
\text { Model } \\
\text { R/ARE } \\
\text { Elovich } \\
\text { B. Constant } \\
\text { (ß) } \\
\end{array}$ & $\begin{array}{c}\text { Webber- } \\
\text { Morris K. E } \\
\text { Model } \\
\text { R}^{2} / \mathrm{ARE} \\
\text { Biosorption } \\
\text { Constant (K) }\end{array}$ & $\begin{array}{l}\text { Ist Proposed } \\
\text { K. Model } \\
\text { R}^{2} / A R E \\
\text { Biosorption } \\
\text { Constant (K) }\end{array}$ & $\begin{array}{c}\text { 2nd Kinetic } \\
\text { K. Model } \\
\text { R}^{2} / A R E \\
\text { Biosorption } \\
\text { Constant (K) }\end{array}$ \\
\hline Bacillus circulans & $\begin{array}{l}0.9982 \\
44.06 \% \\
0.000332\end{array}$ & $\begin{array}{l}0.9481 \\
14.22 \% \\
0.000017\end{array}$ & $\begin{array}{l}0.9982 \\
35.25 \% \\
-0.000332\end{array}$ & $\begin{array}{l}0.9482 \\
54.86 \% \\
0.000023\end{array}$ & $\begin{array}{l}0.9169 \\
21.33 \% \\
0.0791\end{array}$ & $\begin{array}{l}0.9971 \\
3.02 \% \\
1.0859\end{array}$ & $\begin{array}{l}0.9990 \\
1.72 \% \\
0.000451\end{array}$ & $\begin{array}{l}0.9981 \\
2.43 \% \\
0.8725\end{array}$ \\
\hline $\begin{array}{l}\text { Pseudomonas } \\
\text { aeruginosa }\end{array}$ & $\begin{array}{l}0.9966 \\
49.80 \% \\
0.000299\end{array}$ & $\begin{array}{l}0.9638 \\
10.72 \% \\
0.000015\end{array}$ & $\begin{array}{l}0.9966 \\
31.26 \% \\
-0.000299\end{array}$ & $\begin{array}{l}0.9529 \\
64.80 \% \\
0.000030\end{array}$ & $\begin{array}{l}0.9128 \\
14.78 \% \\
0.0886\end{array}$ & $\begin{array}{l}0.9913 \\
4.48 \% \\
0.9688\end{array}$ & $\begin{array}{l}0.9967 \\
2.38 \% \\
0.000406\end{array}$ & $\begin{array}{l}0.9924 \\
4.60 \% \\
0.7785\end{array}$ \\
\hline $\begin{array}{l}\text { Staphylococcus } \\
\text { xylosus }\end{array}$ & $\begin{array}{l}0.6630 \\
63.37 \% \\
0.000548\end{array}$ & $\begin{array}{l}0.6933 \\
83.60 \% \\
0.0000073\end{array}$ & $\begin{array}{l}0.6630 \\
118.76 \% \\
-0.000548\end{array}$ & $\begin{array}{l}0.9996 \\
9.26 \% \\
0.000130\end{array}$ & $\begin{array}{l}0.8942 \\
21.30 \% \\
0.0687\end{array}$ & $\begin{array}{l}0.7091 \\
37.85 \% \\
1.0685 \\
\end{array}$ & $\begin{array}{l}0.6554 \\
11.24 \% \\
0.000688\end{array}$ & $\begin{array}{l}0.6997 \\
38.56 \% \\
0.8524\end{array}$ \\
\hline $\begin{array}{l}\text { Streptomyces } \\
\text { rimosus }\end{array}$ & $\begin{array}{l}0.9235 \\
54.32 \% \\
0.000516\end{array}$ & $\begin{array}{l}0.9945 \\
10.02 \% \\
0.000051\end{array}$ & $\begin{array}{l}0.9235 \\
80.45 \% \\
-0.000516\end{array}$ & $\begin{array}{l}0.9981 \\
10.44 \% \\
0.000052\end{array}$ & $\begin{array}{l}0.9654 \\
16.46 \% \\
0.0659\end{array}$ & $\begin{array}{l}0.8466 \\
39.92 \% \\
1.1711\end{array}$ & $\begin{array}{l}0.9059 \\
5.60 \% \\
0.000663\end{array}$ & $\begin{array}{l}0.8397 \\
40.92 \% \\
0.9366\end{array}$ \\
\hline $\begin{array}{l}\text { Saccharomyces sp. } \\
\text { (Yeast) }\end{array}$ & $\begin{array}{l}0.9965 \\
35.49 \% \\
0.000348\end{array}$ & $\begin{array}{l}0.9376 \\
16.01 \% \\
0.000017\end{array}$ & $\begin{array}{l}0.9965 \\
36.74 \% \\
-0.000348\end{array}$ & $\begin{array}{l}0.9451 \\
28.52 \% \\
0.000015\end{array}$ & $\begin{array}{l}0.9309 \\
35.42 \% \\
0.0707\end{array}$ & $\begin{array}{l}0.9988 \\
3.54 \% \\
1.2069\end{array}$ & $\begin{array}{l}0.9974 \\
0.88 \% \\
0.000476\end{array}$ & $\begin{array}{l}0.9992 \\
4.41 \% \\
0.9694\end{array}$ \\
\hline
\end{tabular}

Table 2: Summary of kinetic equations for biosorption of Zinc 
Table 3: Summary of kinetic equations for biosorption of Copper

\begin{tabular}{|c|c|c|c|c|c|c|c|c|}
\hline Microbe\$ K.Eq. & $\begin{array}{c}1^{\text {st }} \text { Order K. } \\
\text { Model } \\
\mathrm{R}^{2} / \mathrm{ARE} \\
\text { Biosorption } \\
\text { Constant }(\mathrm{K})\end{array}$ & $\begin{array}{c}\text { 2nd Order K. } \\
\text { Equati. } \\
\mathrm{R}^{2} / \mathrm{ARE} \\
\text { Biosorption } \\
\text { Constant (K) }\end{array}$ & $\begin{array}{c}\text { Pseudo } 1^{\text {st_ }} \\
\text { Order K. } \\
\text { Model } \\
\text { R}^{2} / A R E \\
\text { Biosorption } \\
\text { Constant (K) }\end{array}$ & $\begin{array}{l}\text { Pseudo } 2^{\text {nd. }} \\
\text { Order K. } \\
\text { Model } \\
\mathrm{R}^{2} / \mathrm{ARE} \\
\text { Biosorption } \\
\text { Constant (K) }\end{array}$ & $\begin{array}{c}\text { Elovich K. } \\
\text { Model } \\
\text { R}^{2} / A R E \\
\text { Elovich B. } \\
\text { Constant }(\beta)\end{array}$ & $\begin{array}{c}\text { Webber- } \\
\text { Morris K. } \\
\text { Model } \\
\mathrm{R}^{2} / \mathrm{ARE} \\
\text { Biosorption } \\
\text { Constant (K) }\end{array}$ & $\begin{array}{l}\text { Ist Proposed } \\
\text { K. Model } \\
\mathrm{R}^{2} / \mathrm{ARE} \\
\text { Biosorption } \\
\text { Constant (K) }\end{array}$ & $\begin{array}{c}\text { 2nd Kinetic } \\
\text { K. Model } \\
\text { R}^{2} / A R E \\
\text { Biosorption } \\
\text { Constant (K) }\end{array}$ \\
\hline Bacillus circulans & $\begin{array}{c}0.9674 \\
47.62 \% \\
0.000748\end{array}$ & $\begin{array}{c}0.9334 \\
68.03 \% \\
0.000035\end{array}$ & $\begin{array}{c}0.9674 \\
811.98 \% \\
-0.000748\end{array}$ & $\begin{array}{c}0.9988 \\
8.72 \% \\
0.000118\end{array}$ & $\begin{array}{c}0.9798 \\
13.94 \% \\
0.1508\end{array}$ & $\begin{array}{c}0.8660 \\
39.32 \% \\
0.5138\end{array}$ & $\begin{array}{c}0.9502 \\
5.56 \% \\
0.000840\end{array}$ & $\begin{array}{c}0.8589 \\
40.40 \% \\
0.4109\end{array}$ \\
\hline $\begin{array}{c}\text { Pseudomonas } \\
\text { aeruginosa }\end{array}$ & $\begin{array}{c}0.8630 \\
55.45 \% \\
0.000411\end{array}$ & $\begin{array}{c}0.9636 \\
15.14 \% \\
0.000071\end{array}$ & $\begin{array}{c}0.8630 \\
82.27 \% \\
-0.000411\end{array}$ & $\begin{array}{c}0.9981 \\
15.04 \% \\
0.000109 \\
\end{array}$ & $\begin{array}{c}0.9713 \\
22.35 \% \\
0.1637 \\
\end{array}$ & $\begin{array}{c}0.8481 \\
57.45 \% \\
0.4705 \\
\end{array}$ & $\begin{array}{c}0.8486 \\
4.88 \% \\
0.000497 \\
\end{array}$ & $\begin{array}{c}0.8407 \\
58.95 \% \\
0.3762 \\
\end{array}$ \\
\hline $\begin{array}{c}\text { Staphylococcus } \\
\text { xylosus }\end{array}$ & $\begin{array}{c}0.9490 \\
52.09 \% \\
0.000426\end{array}$ & $\begin{array}{c}0.9916 \\
7.39 \% \\
0.000073\end{array}$ & $\begin{array}{c}0.9490 \\
81.31 \% \\
-0.000426\end{array}$ & $\begin{array}{c}0.9941 \\
16.07 \% \\
0.000103\end{array}$ & $\begin{array}{l}0.9945 \\
3.94 \% \\
0.1702\end{array}$ & $\begin{array}{c}0.9421 \\
23.60 \% \\
0.4713\end{array}$ & $\begin{array}{c}0.9397 \\
3.74 \% \\
0.000514\end{array}$ & $\begin{array}{c}0.9372 \\
24.54 \% \\
0.3775\end{array}$ \\
\hline $\begin{array}{l}\text { Streptomyces } \\
\text { rimosus }\end{array}$ & $\begin{array}{c}0.9982 \\
5.35 \% \\
0.000667\end{array}$ & $\begin{array}{c}0.8565 \\
85.68 \% \\
0.000182\end{array}$ & $\begin{array}{c}0.9982 \\
203.65 \% \\
-0.000667\end{array}$ & $\begin{array}{c}0.9968 \\
3.21 \% \\
0.000090\end{array}$ & $\begin{array}{l}0.9514 \\
125.3 \% \\
0.1301\end{array}$ & $\begin{array}{l}0.9918 \\
9.45 \% \\
0.6468\end{array}$ & $\begin{array}{c}0.9998 \\
0.14 \% \\
0.000786\end{array}$ & $\begin{array}{l}0.9902 \\
14.57 \% \\
0.5190\end{array}$ \\
\hline $\begin{array}{c}\text { Saccharomyces } \\
\text { sp. (Yeast) }\end{array}$ & $\begin{array}{c}0.8485 \\
55.24 \% \\
0.000369\end{array}$ & $\begin{array}{c}0.9451 \\
15.85 \% \\
0.000054\end{array}$ & $\begin{array}{c}0.8485 \\
66.36 \% \\
-0.000369\end{array}$ & $\begin{array}{c}0.9993 \\
8.47 \% \\
0.000109\end{array}$ & $\begin{array}{l}0.9907 \\
10.78 \% \\
0.1641\end{array}$ & $\begin{array}{l}0.8843 \\
45.5 \% \\
0.4745\end{array}$ & $\begin{array}{c}0.8372 \\
4.14 \% \\
0.000452\end{array}$ & $\begin{array}{l}0.8770 \\
47.00 \% \\
0.3795 \\
\end{array}$ \\
\hline
\end{tabular}

Table 4: Summary of kinetic equations for biosorption of Manganese

\begin{tabular}{|c|c|c|c|c|c|c|c|c|}
\hline Microbe $\$$ K.Eq. & $\begin{array}{c}1^{\text {st }} \text { Order K. } \\
\text { Model. } \\
\text { R}^{2} / A R E \\
\text { Biosorption } \\
\text { Constant (K) }\end{array}$ & $\begin{array}{c}\text { 2nd Order K. } \\
\text { Model. } \\
\text { RªRE } \\
\text { Biosorption } \\
\text { Constant (K) }\end{array}$ & $\begin{array}{l}\text { Pseudo 1st_ } \\
\text { Order K. } \\
\text { Model } \\
\text { R}^{2} / A R E \\
\text { Biosorption } \\
\text { Constant (K) }\end{array}$ & $\begin{array}{l}\text { Pseudo } 2^{\text {nd_ }} \\
\text { Order K. } \\
\text { Model } \\
\text { R}^{2} / A R E \\
\text { Biosorption } \\
\text { Constant (K) }\end{array}$ & $\begin{array}{c}\text { Elovich K. } \\
\text { Model } \\
\text { R}^{2} / A R E \\
\text { Elovich B. } \\
\text { Constant }(\beta)\end{array}$ & $\begin{array}{c}\text { Webber- } \\
\text { Morris K. } \\
\text { Model } \\
\text { R}^{2} / A R E \\
\text { Biosorption } \\
\text { Constant (K) }\end{array}$ & $\begin{array}{l}\text { Ist Proposed } \\
\text { K. Model. } \\
\text { R/ARE } \\
\text { Biosorption } \\
\text { Constant (K) }\end{array}$ & $\begin{array}{l}\text { 2nd Kinetic } \\
\text { K. Model } \\
\text { R}^{2} / A R E \\
\text { Biosorption } \\
\text { Constant (K) }\end{array}$ \\
\hline Bacillus circulans & $\begin{array}{c}0.9982 \\
33.20 \% \\
0.000506\end{array}$ & $\begin{array}{c}0.9040 \\
38.59 \% \\
0.000038 \\
\end{array}$ & $\begin{array}{c}0.9982 \\
65.21 \% \\
-0.000506\end{array}$ & $\begin{array}{c}0.9670 \\
25.11 \% \\
0.000016\end{array}$ & $\begin{array}{c}0.9514 \\
29.09 \% \\
0.0615\end{array}$ & $\begin{array}{l}0.9974 \\
5.88 \% \\
1.3710 \\
\end{array}$ & $\begin{array}{c}0.9990 \\
1.14 \% \\
0.000668 \\
\end{array}$ & $\begin{array}{l}0.9965 \\
7.32 \% \\
0.8725\end{array}$ \\
\hline $\begin{array}{c}\text { Pseudomonas } \\
\text { aeruginosa }\end{array}$ & $\begin{array}{c}0.9974 \\
29.47 \% \\
0.000335 \\
\end{array}$ & $\begin{array}{c}0.9036 \\
33.40 \% \\
0.000031 \\
\end{array}$ & $\begin{array}{c}0.9974 \\
56.73 \% \\
-0.000812 \\
\end{array}$ & $\begin{array}{c}0.9589 \\
22.57 \% \\
0.000012 \\
\end{array}$ & $\begin{array}{c}0.9395 \\
41.60 \% \\
0.0619\end{array}$ & $\begin{array}{l}0.9992 \\
2.06 \% \\
1.3727 \\
\end{array}$ & $\begin{array}{c}0.9991 \\
0.74 \% \\
0.000625 \\
\end{array}$ & $\begin{array}{l}0.9989 \\
3.39 \% \\
1.1010 \\
\end{array}$ \\
\hline $\begin{array}{c}\text { Staphylococcus } \\
\text { xylosus }\end{array}$ & $\begin{array}{c}0.9933 \\
39.84 \% \\
0.000335\end{array}$ & $\begin{array}{c}0.9269 \\
17.65 \% \\
0.000017\end{array}$ & $\begin{array}{c}0.9933 \\
35.31 \% \\
-0.000335\end{array}$ & $\begin{array}{c}0.9235 \\
58.00 \% \\
0.000019\end{array}$ & $\begin{array}{c}0.8945 \\
25.61 \% \\
0.0784 \\
\end{array}$ & $\begin{array}{l}0.9907 \\
6.15 \% \\
1.1061 \\
\end{array}$ & $\begin{array}{c}0.9959 \\
1.30 \% \\
0.000460\end{array}$ & $\begin{array}{l}0.9926 \\
5.44 \% \\
1.1022\end{array}$ \\
\hline $\begin{array}{l}\text { Streptomyces } \\
\text { rimosus }\end{array}$ & $\begin{array}{c}0.9909 \\
26.41 \% \\
0.000344 \\
\end{array}$ & $\begin{array}{c}0.9930 \\
18.74 \% \\
0.000016 \\
\end{array}$ & $\begin{array}{c}0.9909 \\
35.27 \% \\
-0.000344 \\
\end{array}$ & $\begin{array}{c}0.8495 \\
19.02 \% \\
0.000005\end{array}$ & $\begin{array}{c}0.8694 \\
108.9 \% \\
0.0699 \\
\end{array}$ & $\begin{array}{c}0.9797 \\
32.35 \% \\
1.2512 \\
\end{array}$ & $\begin{array}{c}0.9933 \\
0.34 \% \\
0.000473\end{array}$ & $\begin{array}{c}0.9823 \\
29.20 \% \\
1.0061 \\
\end{array}$ \\
\hline $\begin{array}{c}\text { Saccharomyces } \\
\text { sp. (Yeast) }\end{array}$ & $\begin{array}{c}0.9984 \\
32.34 \% \\
0.000404\end{array}$ & $\begin{array}{c}0.9326 \\
22.05 \% \\
0.000023\end{array}$ & $\begin{array}{c}0.9984 \\
45.29 \% \\
-0.000404\end{array}$ & $\begin{array}{c}0.9482 \\
26.13 \% \\
0.000013\end{array}$ & $\begin{array}{c}0.9312 \\
39.68 \% \\
0.0659 \\
\end{array}$ & $\begin{array}{l}0.9984 \\
2.82 \% \\
1.2955 \\
\end{array}$ & $\begin{array}{c}0.9991 \\
0.80 \% \\
0.000546\end{array}$ & $\begin{array}{l}0.9986 \\
4.05 \% \\
1.0410 \\
\end{array}$ \\
\hline
\end{tabular}

From table 3, judging the kinetics of biosorption using least value of ARE\%, the first proposed kinetic model best described the kinetics for biosorption of copper by all the five microbes. In terms of highest value of $\mathrm{R}^{2}$, pseudo second-order kinetic equation described best the kinetics for bisorption of $\mathrm{Cu}^{2+}$ by Bacillus circulans, Pseudomonas aeruginosa and Saccharomyces $s p$., Elovich kinetic model described best the kinetics for the biosorption of $\mathrm{Cu}^{2+}$ by Staphylococcus xylosus and finally, First proposed kinetic model described best the kinetic mechanisms for the biosorption of $\mathrm{Cu}^{2+}$ by Streptomyces rimosus. From table 4, considering both least values of ARE\% and $\mathrm{R}^{2}$, the first proposed kinetic model described best the kinetics for the biosorption of manganese ion by all the biosorbents except for the biosorption of manganese by Pseudomonas aeruginosa where Webber-Morris model performed better in terms of $\mathrm{R}^{2}$. The above results show that biosorption mechanisms are complex one. For instance, Elovich kinetic model produced the highest value of coefficient of correlation $\mathrm{R}^{2}$ for biosorption of copper by Staphylococcus xylosus showing that the rate controlling step is purely chemisorption.

Also, from the comparative study of the eight kinetic models presented above, based on values of linear regression coefficient of correlation $\left(R^{2}\right)$, the first proposed model accounted for $46.7 \%$, pseudo secondorder kinetic model accounted for $40 \%$ while Elovich, Webber Morris and second proposed kinetic models each accounted for $6.7 \%$ respectively of the entire results for biosorption of the three heavy metals by 
the five micro-organisms. Also, based on the value of average relative error (ARE\%), the first proposed kinetic model accounted for $93.3 \%$ while pseudo second-order kinetic model accounted for $6.7 \%$ of the results studied. From the above results, it is can be deduced that for a bisorption process exceeding 24 hours before reaching equilibrium, the first proposed equation is the best among other kinetic models but for biosorption processes that reach equilibrium within few minutes or hours as the case may be, Pseudo second-order kinetic model is the best among others as confirmed by the biosorption of zinc by Staphylococcus xylosus. Also, pseudo second-order kinetic model has also proven reliable for biosorption processes with longer contact time.

\section{CONCLUSIONS}

Summarily, on the average, the 8 kinetics model used to analyse the kinetics of biosorption of three heavy metals by the five selected micro-organisms produced moderately high values of linear regression coefficient of correlation. This has shown that the mechanisms of the biosorption processes are complex. This study has also shown that studying the kinetics of biosorption should not be limited to only on the linear regression coefficient of correlation. Other parameters like average relative errors should be applied with coefficient of correlation for a better judgment. This study has also shown that in terms of adsorption capacity or biosorption capacity as the case might be, pseudo-second order equation accommodated different mechanisms. In terms of concentration of heavy metals, the first proposed kinetic model proved to accommodate different mechanisms of biosorption.

\section{REFERENCES}

1. Ahalya, N., Ramachandra, T.V. and Kanamadi, R.D. "Biosorption of Heavy Metals," Research Journal Of Chemistry And Environment, Vol. 7, Dec. 2003.

2. Aksu, A.E., Hiscott, R.N., Yasar, D., Isler, F.I., Marsh, S., Seismic stratigraphy of Late Quaternary deposits from the Southwestern Black Sea shelf: Evidence for noncatastrophic variations in sea-level during the last 10 000 years. Mar. Geol. 190, 2002.

3. Tsezos, M. The selective extraction of metals from solution by microorganisms. Candadian Metallurgical Quarterly, 24(2): 141-144, 1985.

4. Darpeau, A.J., Laurence, R. A. Harbec, P.S., SaintGennain, G. and Lambert, N.G.. Science et Techniques de I'Eau, 16(4), 359, 1983.
5. Krishna R. Hema, Comparative Studies of Isotherm and Kinetics on the Adsorption of $\mathrm{Cr}$ (VI) and Ni (II) from Aqueous Solutions by Powder of Mosambi FruitPeelings. International Research Journal of Pure \& Applied Chemistry, 4(1): 26-45, 2014.

6. Bailey, S. E., Olin TJ, Bricka, R. M., and Adrian, D. D. A review of potentially low cost sorbents for heavy metals. Wat. Res. 33:2469-2479, 1999.

7. Burrell, D. C. Atomic Spectrometric Analysis of heavy Metal Pollutants in Water, Ann Arbor, Mich. 89, 1974.

8. Andrès, Y., Texier, A. C. and Cloirec, P. Le Rare earth elements removal by microbial biosorption: a review. Environ. Technol. 24: 1367-1375, 2003.

9. Atkinson, B. W., Bux, F. and Kasan, H. C. Considerations for application of biosorption technology to remediate metal-contaminated industrial effluents. Water SA 24: 129-135, 1998.

10. Malik, A. Metal bioremediation through growing cells. Environ. Int. 30: 261-278, 2004.

11. Gavrilescu, M. Removal of heavy metals from the environment by biosorption. Eng. Life Sci. 4: 219-232, 2004.

12. Modak, J. M. and Natarajan, K. A. Biosorption of metals using nonliving biomass-a review. Miner. Metall. Proc. 12: 189-196. 1995.

13. Vijayaraghavan, K. and Yun, Y. S. Bacterial biosorbents and biosorption. Biotechnol. Adv. 26: 266-291, 2008.

14. Abu-Elella, R., Ossman, M. E., Abd-Elfatah, M. and Elgendy, A.. Kinetic modeling and isotherm study for naphthalene adsorption on boehmite nanopowder., Desalination and Water Treatment Journal, 51, 34723481, 2013.

15. Ho, Y. S. Review of second-order models for adsorption systems. J. Hazard. Mater. 136: 681-689, 2006.

16. Lagergren, S. Zur theorie der sogenannten adsorption gelöster stoffe. K. Sven.b Vetenskapsakad. Handl. 24: 1$39,1898$.

17. Zeldowitsch, J., U* ber den mechanismus der katalytischen oxidation von $\mathrm{CO}$ and $\mathrm{MnO2}$, Acta Physicochim. URSS 1 364-449., 1934.

18. Weber, W. J. and Morris J. C. Kinetics of adsorption on carbon solution. J. Sanit. Eng. Div. Am. Soc. Civ. Eng. 89: 31-59, 1963.

19. Liu, Y. and Y.-J. Liu Biosorption isotherms, kinetics, and thermodynamics. Sep. Purif. Technol. 61: 229-242, 2008.

20. Nwidi, I.C. and Agunwamba, J. C. Kinetics of biosorption of three heavy metals by five free microorganisms. J. Bioremed Biodeg. 7 (2), 1-5, 2016.

21. Kapoor, A. and Yang, R.T. Surface diffusion on energetically heterogeneous surfaces. AIChE J. 35, 1735-1738, 1989. 